\title{
One FIB Does It All: From Massive Ablation to Nanometer Precision
}

\author{
Carl Kübler ${ }^{1}$, Katrin Fladischer ${ }^{1}$, Katrin Schulthei ${ }^{1}$ and Raymond Hill ${ }^{2}$ \\ ${ }^{1}$ Carl Zeiss Microscopy GmbH, Oberkochen, Germany. \\ ${ }^{2}$ Carl Zeiss Microscopy, LLC, Ion Microscopy Innovation Center, Peabody, USA.
}

Since focused ion beam technology has entered the stage just over thirty years ago [1] it has become indispensable in fundamental science studies and technological applications. One major driving force behind the popularity of FIB technology has been that it offers both high-resolution imaging and flexible micromachining in a single platform.

In recent years the demand for larger material removal rates and increased throughput has driven the development of massive ablation technologies such as FIBs based on plasma sources [2]. However, these instruments are limited in their performance at low currents: the microscale virtual source size of the plasma source does not allow to image at high resolution or perform sophisticated patterning tasks at the nanometer scale. In contrast, the combination of a Ga FIB with a microfocused pulsed laser provides access to a much broader application space [3], [4]. Yet the overall speed of the combined laser and FIB workflow will be eventually limited by the maximum available ion current.

At present, commercially available Ga FIB columns support currents in the range of $50 \mathrm{nA}$. Pushing the envelope of $\mathrm{Ga}$ focused ion beam technology beyond this value without compromising the performance at currents down to below $1 \mathrm{pA}$ presents many challenging issues. At low beam currents a column design with minimal chromatic aberration is needed, yet at the higher beam currents the spherical aberration must be minimized. A further consideration has to be for the ion Coulomb interactions that can occur within the beam itself [5]. Minimizing these Coulomb effects adds further constraints for the design, in terms of aperture placement and operating conditions.

In Figure 1 we present the calculated beam performance of a novel column design that is the result of a careful and thorough optimization of all the competing effects. The column delivers currents of up to $100 \mathrm{nA}$ and provides beam current densities in excess of $70 \mathrm{nA} / \mathrm{cm}^{2}$.

The column performs exceptionally well at low currents. Its smallest probe size has been experimentally verified with a statistically averaged edge measurement [6] to be less than $2.5 \mathrm{~nm}$ at $1 \mathrm{pA}$.

The new column design gives access to five orders of magnitude of ion beam current. The complete application space of a conventional Ga FIB (see Figure 2) is covered. For many processes that require the removal of large amounts of material the presented results will essentially reduce the process time by a factor of two compared to current state of the art FIB columns. Yet ultimate precision at the nanometer scale is preserved.

[1] R.L. Seliger et al, Appl. Phys. Lett. 34 (1979), p. 310

[2] P. Tesch et al, EIPBN conference, Portland (2008)

[3] R. Salzer et al, Microsc. Microanal. 18 (Suppl. 2) (2012), p. 636

[4] R. Rosenkranz et al, Imaging \& Microscopy 1 (2012), p. 34

[5] P. Kruit and X. R. Jiang, J. Vac. Sci. Technol. B 14 (1996), p. 3

[6] J. Orloff and L.Y. Roussel, Microsc. Microanal. 16 (Suppl 2) (2010), p. 192 


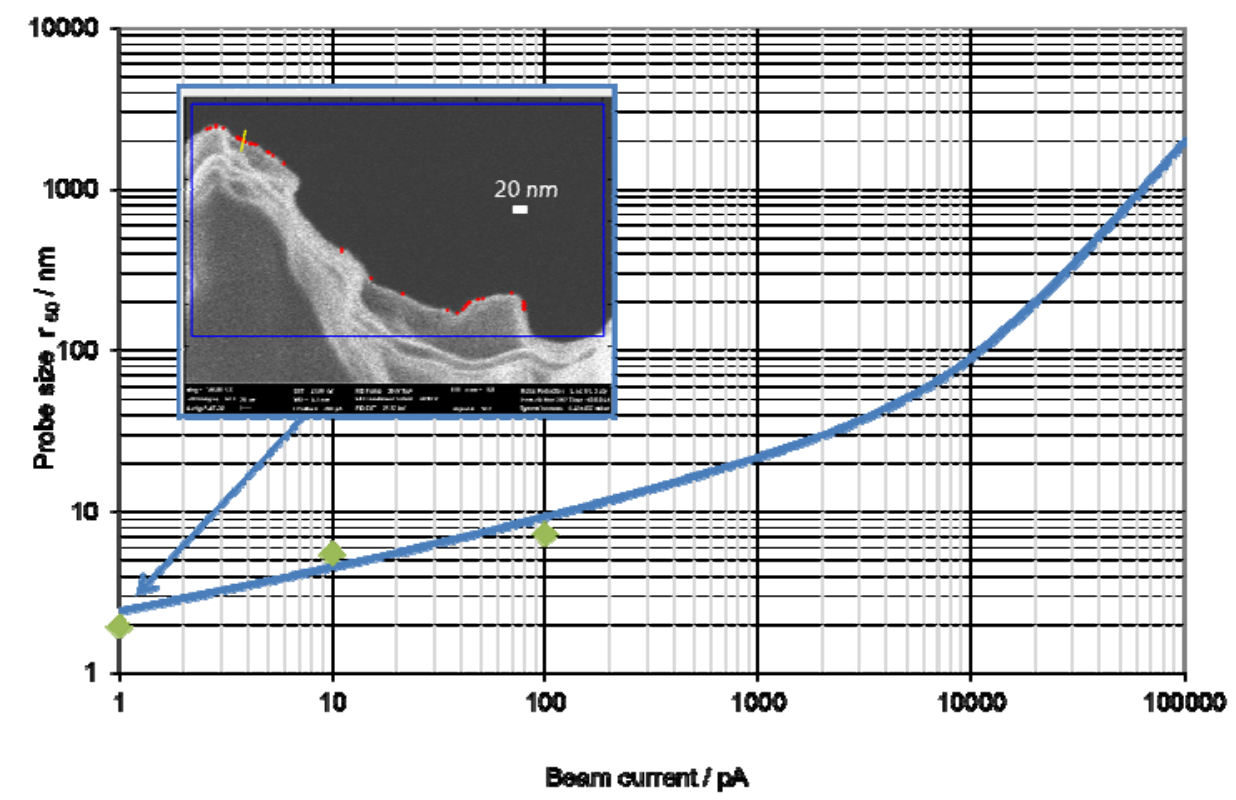

Figure 1. The calculated probe size (r50) at a working distance of $13.5 \mathrm{~mm}$ and an ion energy of $30 \mathrm{kV}$ is shown as the blue curve. Measured resolution values are shown as green data points. The insert displays the measurement at $1 \mathrm{pA}$ : The statistically averaged edge method [6] results in an r50 value of less than $2.5 \mathrm{~nm}$.

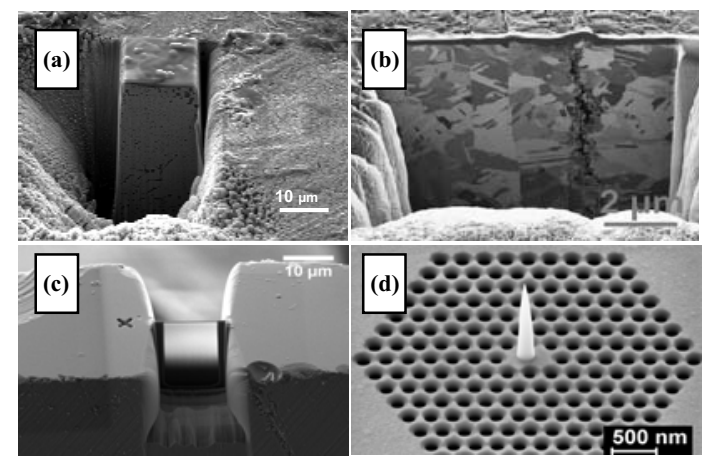

Figure 2. Applications such as (a) 3D EDS analysis (b) preparation of cross-sections and (c) TEM lamella preparation will greatly benefit from the increased current that is maximally available. At the same time no precision is lost and even delicate nanopatterning tasks may be performed (d). 